\title{
Synthesis and Mesomorphic Properties of Tropon-4-yl 4-n-Alkoxybenzoates: Roles of a Tropone Ring as a Polar Terminal Group
}

\author{
Kanji Kubo $^{1 *}$, Akira Mori ${ }^{1}$, Seiji UJIIE ${ }^{2}$ and Carsten Tschierske ${ }^{3}$ \\ ${ }^{I}$ Institute for Materials Chemistry and Engineering, Kyushu University \\ (Kasuga-koen, Kasuga, Fukuoka 816-8580, JAPAN) \\ ${ }^{2}$ Department of Material Science, Interdisciplinary Faculty of Science and \\ Engineering, Shimane University \\ (Nishikawatsu, Matue 690-8504, JAPAN) \\ ${ }^{3}$ Department of Chemistry, Institute of Organic Chemistry, \\ Martin-Luther-University Halle-Wittenberg \\ (Kurt-Mothes Strasse 2, D-06120 Halle/Saale, GERMANY)
}

Edited by A. Masuyama, Osaka Univ., and accepted March 15, 2004 (received for review February 27, 2004)

\begin{abstract}
New troponoid liquid crystals with a tropone terminal group were prepared. Tropon-4-yl 4-n-alkoxybenzoates (1T) had a monotropic smectic A phase. However the corresponding benzenoid derivatives (1B), tropolon-5-yl 4- $n$-alkoxybenzoates $(\mathbf{2 T}), 2$ methoxytropon-5-yl 4-n-alkoxybenzoates (3T), and tropon-2-yl 4- $n$-alkoxybenzoates (4T) were not mesomorphic. X-ray diffraction studies revealed that $\mathbf{1 T}$ formed the interdigitated bilayers in the smectic A phase.
\end{abstract}

Key words: tropone, tropolone, liquid crystal, smectic A phase, bilayer structure

\section{Introduction}

The typical structural units of rod-like liquid crystals possess a fairly rigid core, which generally consists of six-membered ring systems. The preparation of new types of liquid crystals materials with rings other than six-membered rings had received less attention. However, it is important to extend the molecular design of liquid crystals to involve other ring systems form not only theoretical, but also the practical, points of views. Liquid crystals having a polar group such as a cyano group give rise to some interesting behaviors and some of them have successfully proved to be useful electrooptical devices employing the twisted nematic mode $(1,2)$. Tropone and tropolone, seven-membered ring compounds with a carbonyl group, which have the large dipole moments (4.1 and 3.5 D, respectively), which are comparable with that $(4.18 \mathrm{D})$ of benzonitrile $(3,4)$. Hence, tropone and tropolone will be suitable as a terminal group for high stability of liquid crystals. Although previously some liquid crystals with tropolone and 2-methoxytropone as a terminal group have been prepared, they were not mesomorphic (5). However the compounds with a tropone ring as a terminal group have not been reported. In this paper, we will report the syntheses and mesomorphic properties of new liquid crystals (1-4T) with tropone as a terminal group.

\section{Experimental}

\section{2・1 Analysis}

Elemental analyses were performed at the elemental

\footnotetext{
*Correspondence to: Kanji KuBo, Institute for Materials Chemistry and Engineering, Kyushu University, Kasuga-koen, Kasuga, Fukuoka 8168580, JAPAN

E-mail: kubo-k@cm.kyushu-u.ac.jp
} 
analysis laboratory of Kyushu University. The melting points were obtained using a Yanagimoto micro-melting-point apparatus and are uncorrected. The NMR spectra were recorded using JEOL Lambda 400 and 600 spectrometer and solutions in $\mathrm{CDCl}_{3}$ at room temperature; the chemical shifts are expressed in $\delta$ units. The mass spectra were measured with JEOL JMS-700 spectrometers. The transition temperatures were measured by differential scanning calorimetry (Seiko DSC 6200) and the mesomorphic phases were observed by polarizing optical microscopy (Olympus BHSP BH-2 equipped with a Linkam TH-600MS hot stage). X-ray diffraction measurements were carried out with a Rigaku Rint 2100 system using Ni-filtered $\mathrm{Cu}-\mathrm{K} \alpha$ radiation at various temperatures. The measuring temperatures were controlled with a Linkam HFS-91 hot stage.

\section{$2 \cdot 2$ Synthesis}

\section{$2 \cdot 2 \cdot 1$ Preparation of 1T, 2T, and 4T}

Alkoxybenzoyl chloride $(0.6 \mathrm{mmol})$ was added to a pyridine $\left(0.5 \mathrm{~cm}^{3}\right)$ solution of the corresponding tropolones (4-hydroxytropone (6), 5-hydroxytropolone, and tropolone: $0.5 \mathrm{mmol}$ ) and 4,4-dimethylaminopyridne (DMAP: $12.2 \mathrm{mg}, 0.1 \mathrm{mmol}$ ). The reaction mixture was stirred at room temperature for $3 \mathrm{~h}$. The mixture was poured into a saturated $\mathrm{KHSO}_{4}$ solution and extracted with chloroform. The organic layer was washed with a saturated $\mathrm{NaCl}$ solution and dried over $\mathrm{Na}_{2} \mathrm{SO}_{4}$. The organic layer was evaporated in vacuo to leave a residue which was chromatographed in a hexane-ethyl acetate or a hexane-chloroform mixture on a silica-gel column (Wako gel C-300) to give pale yellow crystals. The crystals were recrystallized with hexaneethyl acetate $(1: 1 v / v)$ to give pale yellow crystals.

$1 \mathrm{T8}(85 \%) ; \mathrm{MS}(\mathrm{FAB}) \mathrm{m} / \mathrm{z} 355\left(\mathrm{M}+\mathrm{H}^{+}\right)$. Anal. Found: C, 74.30; $\mathrm{H}, 7.43 \%$. Calcd for $\mathrm{C}_{22} \mathrm{H}_{26} \mathrm{O}_{4}: \mathrm{C}, 74.55 ; \mathrm{H}$, $7.39 \% . \quad{ }^{1} \mathrm{H} \mathrm{NMR}\left(\mathrm{CDCl}_{3}\right): \delta 0.89(3 \mathrm{H}, \mathrm{t}, \mathrm{J}=6.6 \mathrm{~Hz})$, $1.25-1.39(8 \mathrm{H}, \mathrm{m}), 1.47(2 \mathrm{H}$, quit, $\mathrm{J}=6.6 \mathrm{~Hz}), 1.82$ $(2 \mathrm{H}$, quit, $\mathrm{J}=6.6 \mathrm{~Hz}), 4.05(2 \mathrm{H}, \mathrm{t}, \mathrm{J}=6.6 \mathrm{~Hz}), 6.86$ $(1 \mathrm{H}, \mathrm{d}, \mathrm{J}=9.5 \mathrm{~Hz}), 6.97(2 \mathrm{H}, \mathrm{d}, \mathrm{J}=8.8 \mathrm{~Hz}), 7.00-7.10$ $(3 \mathrm{H}, \mathrm{m}), 7.13(1 \mathrm{H}, \mathrm{dd}, \mathrm{J}=12.1,9.5 \mathrm{~Hz}), 8.08(2 \mathrm{H}, \mathrm{d}$, $\mathrm{J}=8.8 \mathrm{~Hz}) .{ }^{13} \mathrm{C} \mathrm{NMR}\left(\mathrm{CDCl}_{3}\right): \delta 14.1,22.7,26.0,29.1$, $29.2,29.3,31.8,68.5,114.5$ (2C), 120.2, 125.0, 132.5 (2C), 134.6, 134.7, 140.6, 141.0, 155.2, 164.1, 164.5, 186.9

$1 \mathrm{T12}(89 \%)$; MS(FAB) m/z $411\left(\mathrm{M}+\mathrm{H}^{+}\right)$. Anal. Found: C, 75.89; $\mathrm{H}, 8.35 \%$. Calcd for $\mathrm{C}_{26} \mathrm{H}_{34} \mathrm{O}_{4}: \mathrm{C}, 76.06 ; \mathrm{H}$, $8.35 \% .{ }^{1} \mathrm{H}$ NMR $\left(\mathrm{CDCl}_{3}\right): \delta 0.88(3 \mathrm{H}, \mathrm{t}, \mathrm{J}=6.6 \mathrm{~Hz})$,
1.27-1.36 (16H, m), $1.47(2 \mathrm{H}$, quit, $\mathrm{J}=6.6 \mathrm{~Hz}), 1.82$ $(2 \mathrm{H}$, quit, $\mathrm{J}=6.6 \mathrm{~Hz}), 4.05(2 \mathrm{H}, \mathrm{t}, \mathrm{J}=6.6 \mathrm{~Hz}), 6.86$ $(1 \mathrm{H}, \mathrm{d}, \mathrm{J}=9.5 \mathrm{~Hz}), 6.97(2 \mathrm{H}, \mathrm{d}, \mathrm{J}=8.8 \mathrm{~Hz}), 7.01-7.09$ $(3 \mathrm{H}, \mathrm{m}), 7.13(1 \mathrm{H}, \mathrm{dd}, \mathrm{J}=12.1,9.5 \mathrm{~Hz}), 8.08(2 \mathrm{H}, \mathrm{d}$, $\mathrm{J}=8.8 \mathrm{~Hz}) .{ }^{13} \mathrm{C} \mathrm{NMR}\left(\mathrm{CDCl}_{3}\right): \delta 14.1,22.6,25.9,29.0$, 29.3, 29.5 (2C), 29.7, 29.6 (2C), 31.9, 68.4, 114.5 (2C), $120.2,125.1,132.4$ (2C), 134.6, 134.7, 140.5, 141.0, $155.2,164.1,164.4,186.9$.

$1 \mathrm{T16}(82 \%)$; MS(FAB) m/z $467\left(\mathrm{M}+\mathrm{H}^{+}\right)$. Anal. Found: C, 76.92; H, 9.07\%. Calcd for $\mathrm{C}_{30} \mathrm{H}_{42} \mathrm{O}_{4}: \mathrm{C}, 77.21 ; \mathrm{H}$, 9.07\%. ${ }^{1} \mathrm{H} \mathrm{NMR}\left(\mathrm{CDCl}_{3}\right): \delta 0.88(3 \mathrm{H}, \mathrm{t}, \mathrm{J}=6.6 \mathrm{~Hz})$, 1.26-1.38 (24H, m), $1.47(2 \mathrm{H}$, quit, $\mathrm{J}=6.6 \mathrm{~Hz}), 1.82$ $(2 \mathrm{H}$, quit, $\mathrm{J}=6.6 \mathrm{~Hz}), 4.05(2 \mathrm{H}, \mathrm{t}, \mathrm{J}=6.6 \mathrm{~Hz}), 6.86$ $(1 \mathrm{H}, \mathrm{d}, \mathrm{J}=9.5 \mathrm{~Hz}), 6.97(2 \mathrm{H}, \mathrm{d}, \mathrm{J}=8.8 \mathrm{~Hz}), 7.01-7.09$ $(3 \mathrm{H}, \mathrm{m}), 7.13(1 \mathrm{H}, \mathrm{dd}, \mathrm{J}=12.1,9.5 \mathrm{~Hz}), 8.08(2 \mathrm{H}, \mathrm{d}$, $\mathrm{J}=8.8 \mathrm{~Hz}) .{ }^{13} \mathrm{C} \mathrm{NMR}\left(\mathrm{CDCl}_{3}\right): \delta 14.1,29.1,29.3,29.4$, 29.55, 29.59 (2C), 29.65 (2C), 29.68 (2C), 29.69 (3C), 31.9, 68.5, 114.5 (2C), 120.2, 125.1, 132.5 (2C), 134.6, 134.7, 140.6, 141.0, 155.2, 164.0, 164.4, 186.9 . $2 T 8$ (47\%); MS(FAB) m/z $371\left(\mathrm{M}+\mathrm{H}^{+}\right)$. Anal. Found: C, 71.29; H, 7.08\%. Calcd for $\mathrm{C}_{22} \mathrm{H}_{26} \mathrm{O}_{5}: \mathrm{C}, 71.33 ; \mathrm{H}$, $7.07 \% .{ }^{1} \mathrm{H} \mathrm{NMR}\left(\mathrm{CDCl}_{3}\right): \delta 0.90(3 \mathrm{H}, \mathrm{t}, \mathrm{J}=6.6 \mathrm{~Hz})$, $1.25-1.38(8 \mathrm{H}, \mathrm{m}), 1.48(2 \mathrm{H}$, quit, $\mathrm{J}=6.6 \mathrm{~Hz}), 1.83$ $(2 \mathrm{H}$, quit, $\mathrm{J}=6.6 \mathrm{~Hz}), 4.05(2 \mathrm{H}, \mathrm{t}, \mathrm{J}=6.6 \mathrm{~Hz}), 6.98$ $(1 \mathrm{H}, \mathrm{d}, \mathrm{J}=8.8 \mathrm{~Hz}), 7.27(2 \mathrm{H}, \mathrm{d}, \mathrm{J}=12.0 \mathrm{~Hz}), 7.34(1 \mathrm{H}$, $\mathrm{d}, \mathrm{J}=12.0 \mathrm{~Hz}), 8.10(2 \mathrm{H}, \mathrm{d}, \mathrm{J}=8.8 \mathrm{~Hz}) .{ }^{13} \mathrm{C} \mathrm{NMR}$ $\left(\mathrm{CDCl}_{3}\right): \delta 14.1,22.7,26.0,29.1,29.2,29.3,31.8,68.4$, $114.5,120.4,122.6,132.0,132.4,154.1,164.0,165.0$, 170.8

$2 \mathrm{T12}(52 \%)$; MS(FAB) m/z $427\left(\mathrm{M}+\mathrm{H}^{+}\right)$. Anal. Found: C, 72.93; H, 8.02\%. Calcd for $\mathrm{C}_{26} \mathrm{H}_{34} \mathrm{O}_{5}: \mathrm{C}, 73.21 ; \mathrm{H}$, $8.03 \%$. ${ }^{1} \mathrm{H}$ NMR $\left(\mathrm{CDCl}_{3}\right): \delta 0.88(3 \mathrm{H}, \mathrm{t}, \mathrm{J}=6.6 \mathrm{~Hz})$, $1.27-1.38(16 \mathrm{H}, \mathrm{m}), 1.48(2 \mathrm{H}$, quit, $\mathrm{J}=6.6 \mathrm{~Hz}), 1.83$ $(2 \mathrm{H}$, quit, $\mathrm{J}=6.6 \mathrm{~Hz}), 4.05(2 \mathrm{H}, \mathrm{t}, \mathrm{J}=6.6 \mathrm{~Hz}), 6.98$ $(1 \mathrm{H}, \mathrm{d}, \mathrm{J}=8.8 \mathrm{~Hz}), 7.27(2 \mathrm{H}, \mathrm{d}, \mathrm{J}=12.0 \mathrm{~Hz}), 7.34(1 \mathrm{H}$, $\mathrm{d}, \mathrm{J}=12.0 \mathrm{~Hz}), 8.10(2 \mathrm{H}, \mathrm{d}, \mathrm{J}=8.8 \mathrm{~Hz})$.

$2 \mathrm{~T} 16$ (54\%); MS(FAB) m/z $483\left(\mathrm{M}+\mathrm{H}^{+}\right)$. Anal. Found: C, 74.44; $\mathrm{H}, 8.76 \%$. Calcd for $\mathrm{C}_{30} \mathrm{H}_{42} \mathrm{O}_{5}: \mathrm{C}, 74.65 ; \mathrm{H}$, $8.77 \% .{ }^{1} \mathrm{H}$ NMR $\left(\mathrm{CDCl}_{3}\right): \delta 0.88(3 \mathrm{H}, \mathrm{t}, \mathrm{J}=6.6 \mathrm{~Hz})$, $1.26-1.37(24 \mathrm{H}, \mathrm{m}), 1.48(2 \mathrm{H}$, quit, $\mathrm{J}=6.6 \mathrm{~Hz}), 1.83$ $(2 \mathrm{H}$, quit, $\mathrm{J}=6.6 \mathrm{~Hz}), 4.05(2 \mathrm{H}, \mathrm{t}, \mathrm{J}=6.6 \mathrm{~Hz}), 6.98$ $(1 \mathrm{H}, \mathrm{d}, \mathrm{J}=8.8 \mathrm{~Hz}), 7.27(2 \mathrm{H}, \mathrm{d}, \mathrm{J}=12.0 \mathrm{~Hz}), 7.34(1 \mathrm{H}$, $\mathrm{d}, \mathrm{J}=12.0 \mathrm{~Hz}), 8.09(2 \mathrm{H}, \mathrm{d}, \mathrm{J}=8.8 \mathrm{~Hz})$.

4T8 (88\%); MS(FAB) m/z $355\left(\mathrm{M}+\mathrm{H}^{+}\right)$. Anal. Found: C, 74.25; H, 7.34\%. Calcd for $\mathrm{C}_{22} \mathrm{H}_{26} \mathrm{O}_{4}$ : C, 74.55; H, 7.39\%. ${ }^{1} \mathrm{H}$ NMR $\left(\mathrm{CDCl}_{3}\right): \delta 0.89(3 \mathrm{H}, \mathrm{t}, \mathrm{J}=6.6 \mathrm{~Hz})$, $1.27-1.38(8 \mathrm{H}, \mathrm{m}), 1.47(2 \mathrm{H}$, quit, $\mathrm{J}=6.6 \mathrm{~Hz}), 1.82$ $(2 \mathrm{H}$, quit, $\mathrm{J}=6.6 \mathrm{~Hz}), 4.03(2 \mathrm{H}, \mathrm{t}, \mathrm{J}=6.6 \mathrm{~Hz}), 6.96$ 
$(2 \mathrm{H}, \mathrm{d}, \mathrm{J}=8.8 \mathrm{~Hz}), 7.06-7.43(5 \mathrm{H}, \mathrm{m}), 8.12(2 \mathrm{H}, \mathrm{d}, \mathrm{J}=$ $8.8 \mathrm{~Hz})$.

4T12 (89\%); MS(FAB) m/z $411\left(\mathrm{M}+\mathrm{H}^{+}\right)$. Anal. Found: C, 76.03; H, 8.32\%. Calcd for $\mathrm{C}_{26} \mathrm{H}_{34} \mathrm{O}_{4}: \mathrm{C}, 76.06 ; \mathrm{H}$, $8.35 \%$. ${ }^{1} \mathrm{H} \mathrm{NMR}\left(\mathrm{CDCl}_{3}\right): \delta 0.88(3 \mathrm{H}, \mathrm{t}, \mathrm{J}=6.6 \mathrm{~Hz})$, $1.28-1.38(16 \mathrm{H}, \mathrm{m}), 1.47(2 \mathrm{H}$, quit, $\mathrm{J}=6.6 \mathrm{~Hz}), 1.82$ $(2 \mathrm{H}$, quit, $\mathrm{J}=6.6 \mathrm{~Hz}), 4.03(2 \mathrm{H}, \mathrm{t}, \mathrm{J}=6.6 \mathrm{~Hz}), 6.96$ $(2 \mathrm{H}, \mathrm{d}, \mathrm{J}=8.8 \mathrm{~Hz}), 7.06-7.43(5 \mathrm{H}, \mathrm{m}), 8.12(2 \mathrm{H}, \mathrm{d}, \mathrm{J}=$ $8.8 \mathrm{~Hz})$.

$4 \mathrm{T16}(90 \%)$; MS(FAB) m/z $467\left(\mathrm{M}+\mathrm{H}^{+}\right)$. Anal. Found: C, 77.34; H, 9.03\%. Calcd for $\mathrm{C}_{30} \mathrm{H}_{42} \mathrm{O}_{4}: \mathrm{C}, 77.21 ; \mathrm{H}$, 9.07\%. ${ }^{1} \mathrm{H} \mathrm{NMR}\left(\mathrm{CDCl}_{3}\right): \delta 0.88(3 \mathrm{H}, \mathrm{t}, \mathrm{J}=6.6 \mathrm{~Hz})$, 1.26-1.37 $(24 \mathrm{H}, \mathrm{m}), 1.47(2 \mathrm{H}$, quit, $\mathrm{J}=6.6 \mathrm{~Hz}), 1.82$ $(2 \mathrm{H}$, quit, $\mathrm{J}=6.6 \mathrm{~Hz}), 4.03(2 \mathrm{H}, \mathrm{t}, \mathrm{J}=6.6 \mathrm{~Hz}), 6.96$ $(2 \mathrm{H}, \mathrm{d}, \mathrm{J}=8.8 \mathrm{~Hz}), 7.06-7.43(5 \mathrm{H}, \mathrm{m}), 8.12(2 \mathrm{H}, \mathrm{d}, \mathrm{J}=$ $8.8 \mathrm{~Hz})$.

\section{$2 \cdot 2 \cdot 2$ Preparation of $\mathbf{3 T}$}

A THF solution $\left(10 \mathrm{~cm}^{3}\right)$ of tropolon-5-yl 4- $n$ alkoxybenzoates $(\mathbf{2 T}, 0.2 \mathrm{mmol})$ was treated with an ethereal solution $\left(10 \mathrm{~cm}^{3}\right)$ of $\mathrm{CH}_{2} \mathrm{~N}_{2}$. After $12 \mathrm{hr}$, the mixture was evaporated in vacuo to leave a residue, which was chromatographed in a hexane-ethyl acetate or a hexane-chloroform mixture on a silica-gel column to give crystals. The crystals were recrystallized with hexane-ethyl acetate $(1: 1 v / v)$ to give pale yellow crystals.

$3 T 8$ (90\%); MS(FAB) m/z $385\left(\mathrm{M}+\mathrm{H}^{+}\right)$. Anal. Found: C, 71.70; H, 7.28\%. Calcd for $\mathrm{C}_{23} \mathrm{H}_{28} \mathrm{O}_{5}: \mathrm{C}, 71.85 ; \mathrm{H}$,
7.34\%. ${ }^{1} \mathrm{H}$ NMR $\left(\mathrm{CDCl}_{3}\right): \delta 0.89(3 \mathrm{H}, \mathrm{t}, \mathrm{J}=6.6 \mathrm{~Hz})$, $1.25-1.38(8 \mathrm{H}, \mathrm{m}), 1.48(2 \mathrm{H}$, quit, $\mathrm{J}=6.6 \mathrm{~Hz}), 1.82$ $(2 \mathrm{H}$, quit, $\mathrm{J}=6.6 \mathrm{~Hz}), 3.97(3 \mathrm{H}, \mathrm{s}), 4.05(2 \mathrm{H}, \mathrm{t}, \mathrm{J}=6.6$ $\mathrm{Hz}), 6.70(1 \mathrm{H}, \mathrm{d}, \mathrm{J}=11.0 \mathrm{~Hz}), 6.94(1 \mathrm{H}, \mathrm{dd}, \mathrm{J}=11.0$, $2.7 \mathrm{~Hz}), 6.98(2 \mathrm{H}, \mathrm{d}, \mathrm{J}=8.8 \mathrm{~Hz}), 7.16(1 \mathrm{H}, \mathrm{dd}, \mathrm{J}=$ 13.0, $2.7 \mathrm{~Hz}), 7.26(1 \mathrm{H}, \mathrm{d}, \mathrm{J}=13.0 \mathrm{~Hz}), 8.10(2 \mathrm{H}, \mathrm{d}$, $\mathrm{J}=8.8 \mathrm{~Hz}) .{ }^{13} \mathrm{C}$ NMR $\left(\mathrm{CDCl}_{3}\right): \delta 14.1,22.7,26.0,29.1$, 29.2, 29.3, 31.8, 56.4, 68.4, 110.4, 114.5 (2C), 120.5, $123.6,132.4$ (2C), 134.0, 136.4, 149.5, 164.0, 164.2, 164.9, 179.6 .

$3 \mathrm{T12}(95 \%)$; MS(FAB) m/z $441\left(\mathrm{M}+\mathrm{H}^{+}\right)$. Anal. Found: C, 73.81; H, 8.14\%. Calcd for $\mathrm{C}_{27} \mathrm{H}_{36} \mathrm{O}_{5}: \mathrm{C}, 73.61 ; \mathrm{H}$, 8.24\%. ${ }^{1} \mathrm{H}$ NMR $\left(\mathrm{CDCl}_{3}\right): \delta 0.88(3 \mathrm{H}, \mathrm{t}, \mathrm{J}=6.6 \mathrm{~Hz})$, 1.27-1.37 (16H, m), $1.48(2 \mathrm{H}$, quit, $\mathrm{J}=6.6 \mathrm{~Hz}), 1.82$ $(2 \mathrm{H}$, quit, $\mathrm{J}=6.6 \mathrm{~Hz}), 3.97(3 \mathrm{H}, \mathrm{s}), 4.05(2 \mathrm{H}, \mathrm{t}, \mathrm{J}=6.6$ $\mathrm{Hz}), 6.70(1 \mathrm{H}, \mathrm{d}, \mathrm{J}=11.0 \mathrm{~Hz}), 6.94(1 \mathrm{H}, \mathrm{dd}, \mathrm{J}=11.0$, $2.7 \mathrm{~Hz}), 6.98(2 \mathrm{H}, \mathrm{d}, \mathrm{J}=8.8 \mathrm{~Hz}), 7.16(1 \mathrm{H}, \mathrm{dd}, \mathrm{J}=$ 13.0, $2.7 \mathrm{~Hz}), 7.26(1 \mathrm{H}, \mathrm{d}, \mathrm{J}=13.0 \mathrm{~Hz}), 8.10(2 \mathrm{H}, \mathrm{d}$, $\mathrm{J}=8.8 \mathrm{~Hz})$.

$3 T 16(92 \%)$; MS(FAB) m/z $497\left(\mathrm{M}+\mathrm{H}^{+}\right)$. Anal. Found: C, 74.79; H, 8.91\%. Calcd for $\mathrm{C}_{31} \mathrm{H}_{44} \mathrm{O}_{5}: \mathrm{C}, 74.96 ; \mathrm{H}$, $8.93 \%$. ${ }^{1} \mathrm{H}$ NMR $\left(\mathrm{CDCl}_{3}\right): \delta 0.88(3 \mathrm{H}, \mathrm{t}, \mathrm{J}=6.6 \mathrm{~Hz})$, 1.26-1.36 (24H, m), 1.48 (2H, quit, $\mathrm{J}=6.6 \mathrm{~Hz}), 1.82$ $(2 \mathrm{H}$, quit, $\mathrm{J}=6.6 \mathrm{~Hz}), 3.97(3 \mathrm{H}, \mathrm{s}), 4.05(2 \mathrm{H}, \mathrm{t}, \mathrm{J}=6.6$ $\mathrm{Hz}), 6.70(1 \mathrm{H}, \mathrm{d}, \mathrm{J}=11.0 \mathrm{~Hz}), 6.94(1 \mathrm{H}, \mathrm{dd}, \mathrm{J}=11.0$, $2.7 \mathrm{~Hz}), 6.98(2 \mathrm{H}, \mathrm{d}, \mathrm{J}=8.8 \mathrm{~Hz}), 7.16(1 \mathrm{H}, \mathrm{dd}, \mathrm{J}=$ 13.0, $2.7 \mathrm{~Hz}), 7.26(1 \mathrm{H}, \mathrm{d}, \mathrm{J}=13.0 \mathrm{~Hz}), 8.10(2 \mathrm{H}, \mathrm{d}, \mathrm{J}$ $=8.8 \mathrm{~Hz}$ ).
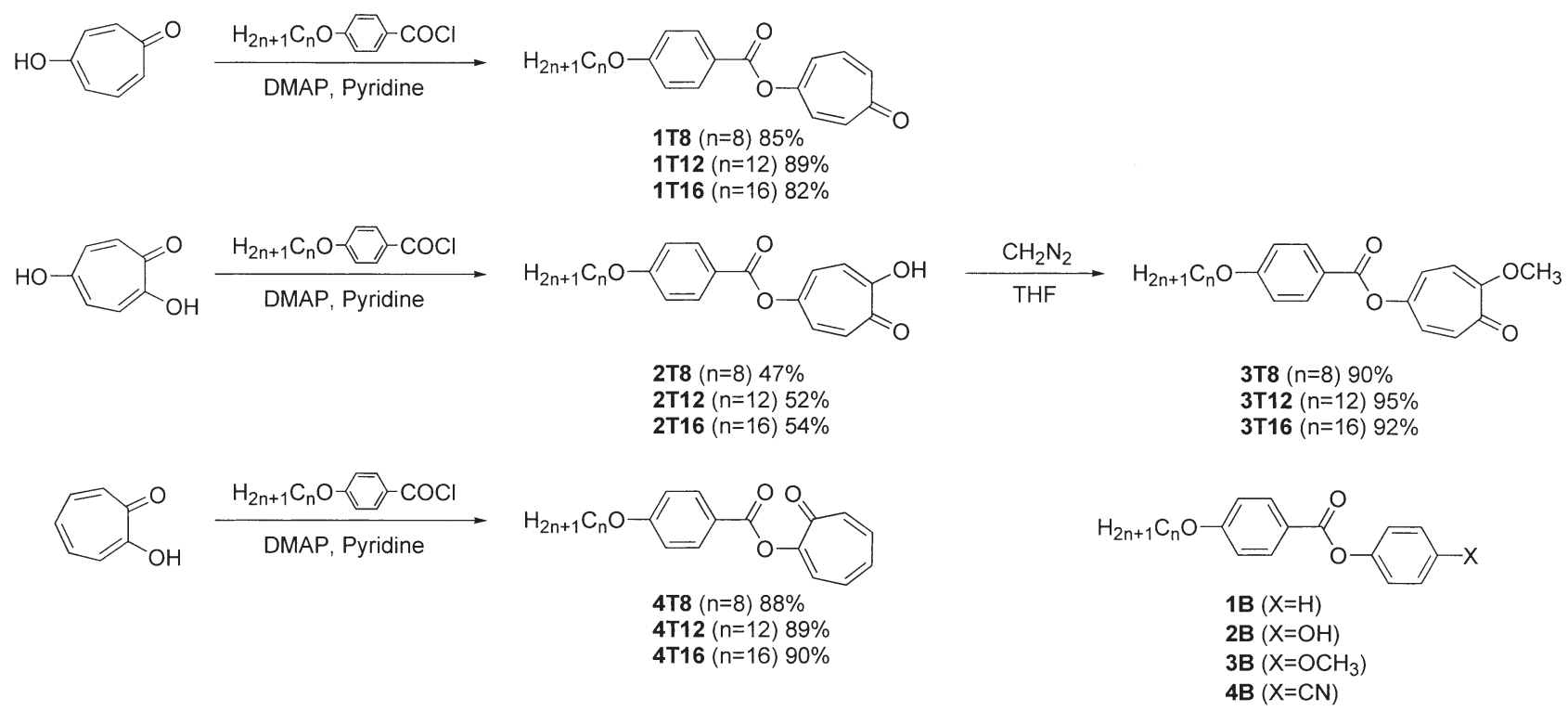

Scheme 


\section{Results and Discussion}

\section{$3 \cdot 1$ Synthesis of Troponoid Compounds}

Tropon-4-yl 4-alkoxybenzoates (1T) and 5-(4alkoxybenzoyloxy)tropolones (2T), 2-(4-alkoxybenzoyloxy)tropolones (4T) were prepared by the esterification of 4-hydroxytropone, 5-hydroxytropolone, and tropolone with 4-alkoxybenzoyl chloride. Methylation of 2T with diazomethane afforded 5-(4-alkoxybenzoyloxy)-2-metoxytropones (3T). The structure and purity of the compounds (1-4) were ascertained by NMR spectroscopy, FAB MS and elemental analysis.

\section{3·2 Mesomorphic Properties}

The transition temperatures and thermal behaviors of the compounds (1-4) were determined using a differential scanning calorimeter and a polarizing microscope equipped with a hot stage as well as an X-ray diffraction study.

Optical micrograph and conoscopic figure of $\mathbf{1 T 1 2}$ are shown in Figs. 1 and 2. The smectic A phase was determined by observation of the bâtonnets, focal-conic fan, and homeotropic textures under a polarzing microscope. When a Bertrand lens was used in the observation of homeotropic texture of 1T12, a dark cross could be seen in the conoscopic figure. This means that 1T align perpendicularly to the layers.

The thermal behaviors of the troponoids (1-4T) and the corresponding benzenoids (1-4B) (7) are summarized in Table 1. The tropone derivatives (1T) have a

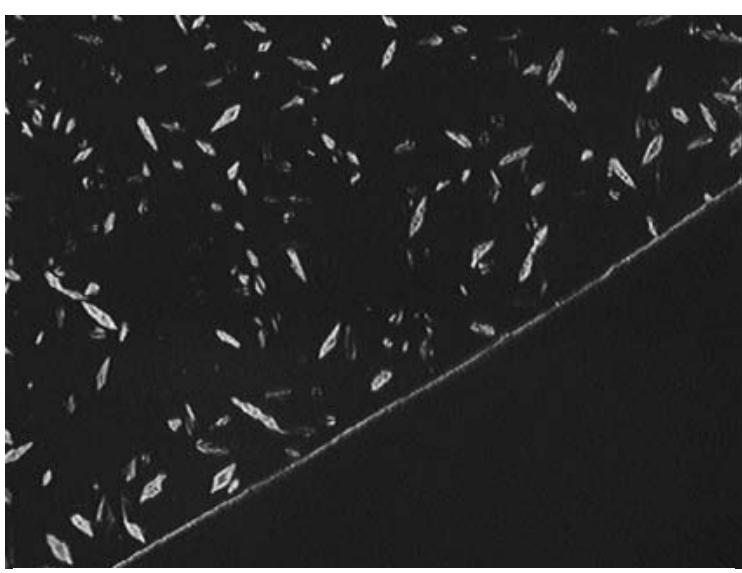

Fig. 1 Texture of the Smectic A Phase of 1T12 at $38^{\circ} \mathrm{C}$ Obtained on Cooling from the Isotropic Liquid.

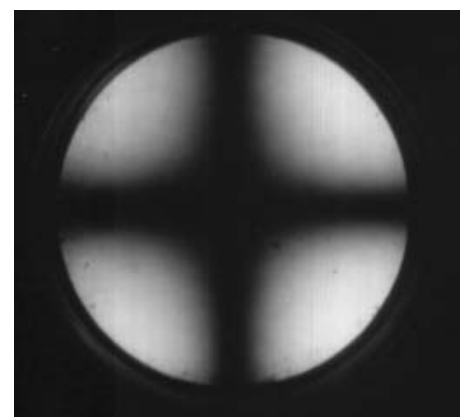

Fig. 2 Conoscopic Figure of $\mathbf{1 T 1 2}$ at $38^{\circ} \mathrm{C}$ Obtained on Cooling from the Isotropic Liquid.

Table 1 Transition Temperatures $\left({ }^{\circ} \mathrm{C}\right)$ of $\mathbf{1 - 4}$.

\begin{tabular}{lrllrl}
\hline & n & Transition Temperatures & & $\mathrm{n}$ & Transition Temperatures \\
\hline $\mathbf{1 T 8}$ & 8 & $\mathrm{Cr} \cdot 37.0 \cdot(\mathrm{SmA} \cdot 2.6 \cdot) \mathrm{I}$ & $\mathbf{1 B 8}^{7}$ & 8 & $\mathrm{Cr} \cdot 59.7 \cdot \mathrm{I}$ \\
$\mathbf{1 T 1 2}$ & 12 & $\mathrm{Cr} \cdot 63.4 \cdot(\mathrm{SmA} \cdot 39.8 \cdot) \mathrm{I}$ & $\mathbf{1 B 1 2}^{2}$ & 12 & $\mathrm{Cr} \cdot 79.3 \cdot \mathrm{I}$ \\
$\mathbf{1 T 1 6}$ & 16 & $\mathrm{Cr} \cdot 71.4 \cdot(\mathrm{SmA} \cdot 63.0 \cdot) \mathrm{I}$ & $\mathbf{1 B 1 6}^{2}$ & 16 & $\mathrm{Cr} \cdot 72.8 \cdot \mathrm{I}$ \\
$\mathbf{2 T 8}$ & 8 & $\mathrm{Cr} \cdot 124.9 \cdot \mathrm{I}$ & $\mathbf{2 B 8}^{7}$ & 8 & $\mathrm{Cr} \cdot 111 \cdot \mathrm{I}$ \\
$\mathbf{2 T 1 2}$ & 12 & $\mathrm{Cr} \cdot 115.2 \cdot \mathrm{I}$ & $\mathbf{2 B 1 2}^{7}$ & 12 & $\mathrm{Cr} \cdot 113 \cdot \mathrm{I}$ \\
TT16 & 16 & $\mathrm{Cr} \cdot 114.8 \cdot \mathrm{I}$ & $\mathbf{2 B 1 6}^{7}$ & 16 & $\mathrm{Cr} \cdot 114.8 \cdot \mathrm{I}$ \\
3T8 & 8 & $\mathrm{Cr} \cdot 90.6 \cdot \mathrm{I}$ & $\mathbf{3 B 8}^{7}$ & 8 & $\mathrm{Cr} \cdot 82 \cdot(\mathrm{N} \cdot 76 \cdot) \mathrm{I}$ \\
3T12 & 12 & $\mathrm{Cr} \cdot 110.6 \cdot \mathrm{I}$ & $\mathbf{3 B 1 2}^{7}$ & 12 & $\mathrm{Cr} \cdot 91 \cdot(\mathrm{N} \cdot 75 \cdot) \mathrm{I}$ \\
3T16 & 16 & $\mathrm{Cr} \cdot 110.7 \cdot \mathrm{I}$ & $\mathbf{3 B 1 6}^{7}$ & 16 & $\mathrm{Cr} \cdot 84 \cdot \mathrm{SmA} \cdot 101 \cdot \mathrm{I}$ \\
4T8 & 8 & $\mathrm{Cr} \cdot 73.3 \cdot \mathrm{I}$ & $\mathbf{4 B 8}^{7}$ & 8 & $\mathrm{Cr} \cdot 75.6 \cdot \mathrm{N} \cdot 88.0 \cdot \mathrm{I}$ \\
4T12 & 12 & $\mathrm{Cr} \cdot 84.4 \cdot \mathrm{I}$ & $\mathbf{4 B 1 2}^{7}$ & 12 & $\mathrm{Cr} \cdot 72.7 \cdot \mathrm{SmA} \cdot 90.0 \cdot \mathrm{I}$ \\
4T16 & 16 & $\mathrm{Cr} \cdot 93.0 \cdot \mathrm{I}$ & $\mathbf{4 B 1 6}^{7}$ & 16 & $\mathrm{Cr} \cdot 83.2 \cdot \mathrm{SmA} \cdot 96.3 \cdot \mathrm{I}$
\end{tabular}

Cr: Crystals, SmA: Smectic A Phase, N: Nematic Phase, I: Isotropic Liquid. 

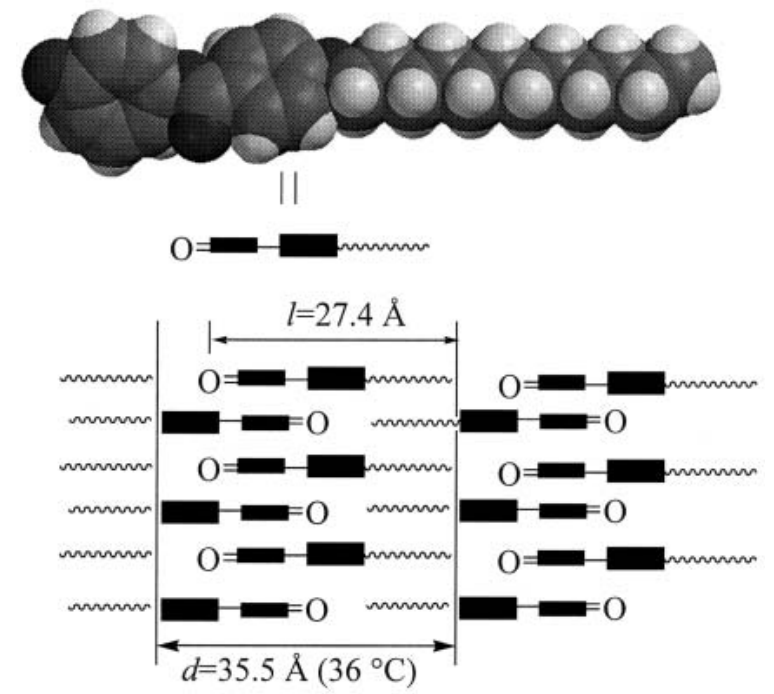

Fig. 3 Packing Model of 1T12 in the Smectic A Phase and Molecular Structure of 1 T12 Generated by Sybyl Calculation.

monotropic smectic A phase, while the corresponding benzenoids (1B) and troponoid derivatives (2T-4T) were not mesomorphic. However the clearing and melting points of the tropone derivatives (1T) were lower than those of 4-cyanophenyl 4-alkanoyloxybenzoate (4B). This is due to the wider molecular width of the seven-membered ring compared with the benzene ring.

\section{3·3 X-Ray Diffraction Analysis}

Furthermore, we measured the X-ray diffraction pattern of the mesophases. The smectic layer spacing $(d)$ of $1 \mathrm{~T} 12$ were observed to be $35.5 \AA\left(36^{\circ} \mathrm{C}\right)$. The molecular length $(l)$ of $\mathbf{1 T 1 2}$ is $27.4 \AA$ (calculated by the Sybyl method of Spartan 04 (8)). The $d / l$ ratio of the smectic A phase of $\mathbf{1 T 1 2}$ are 1.3. This result suggests that $\mathbf{1 T 1 2}$ formed the interdigitated bilayers $(9,10)$ in the smectic A phase as shown in Fig. 3. The $d / l$ value of $\mathbf{1 T}$ was similar to those (1.4 and 1.3) of 4cyanophenyl 4-alkoxybenzoate (7) and 2-(4-alkoxybenzoyl)-5-cyanotropone (9).

\section{Conclusion}

The tropon-4-yl 4-alkoxybenzoates (1T) had a monotropic simectic A phase. They formed the interdigitated bilayers. The 4-hydroxytropone could be used as a terminal group of a mesogenic unit.

\section{Acknowledgements}

This work was supported by the funds from Fuji Photo Film Co. Ltd and Ministry of Education, Science, Sports and Culture of Japan.

\section{References}

1. D. DEMUS, J. GOODBY, G. W. GRAY, H.-W. SPIESS and V. VILL, Handbook of Liquid Crystals, VCH, Weinheim (1998).

2. G.W. GRAY and J.W. GOODBY, Smectic Liquid Crystals. Textures and Structures, Heyden \& Son Inc, Philadelphia (1984).

3. M. KUBO, T. NOZOE and K. KURITA, Dipole Moments of Tropolone and Related Compounds, Nature, Vol. 167, 688-689 (1951).

4. R.D. LIDE, Handbook of Chemistry and Physics, 81st. Ed., CRC Press, Boston, USA (2000).

5. T. UEMURA, S. TAKENAKA, S. KUSABAYASHI and S. SETO, Syntheses and Thermal Properties of New Liquid Crystalline Materials Involving Tropolone, Mol. Cryst. Liq. Cryst., Vol. 95, 287-297 (1983).

6. Y. IKEDA, A. MORI and H. TAKESHITA, An Improved Synthesis of 4-Hydroxy-2,4,6-Cycloheptatrien-1-One (4-Hydroxytropone), Bull. Chem. Soc. Jpn., Vol. 66, 2779-2780 (1993).

7. V. Vill, LiqCryst 4.4-Database of Liquid Crystalline Compounds, Fujitsu Kyushu System Engineering (FQS), Fukuoka (2003).

8. W. J. HEHRE, SPARTAN `04, Wavefunction Inc., USA (2003).

9. M. HASHIMOTO, S. UJIIE and A. MORI, Liquid Crystals with a 5-Cyanotropone Skeleton: 2-(4-Alkoxybenzoyloxy)-5-Cyanotropones and 2-(4-Alkoxybenzoylamino)-5-Cyanotropones, Chem. Lett., 758-759 (2000).

10. K. KUBO, T. TSURUTA, M. HASHIMOTO, A. MORI and S. UJIIE, Synthesis, Crystal Structure, and Mesomorphic Properties of Liquid Crystals with a Nitrotropone Skeleton, ITE Lett. on Batteries, New Tech. \& Med., Vol. 3 (4), 475-478 (2002). 